Check for updates

Cite this: RSC Adv., 2017, 7, 47681

\title{
Efficient and straightforward click synthesis of structurally related dendritic triazoles $\uparrow$
}

\author{
M. I. Mangione, (D)* R. A. Spanevello (D) and M. B. Anzardi \\ A simple, rapid and efficient copper-catalyzed 1,3-dipolar cycloaddition reaction is described for the \\ synthesis of a novel family of twelve triazolic dendrimers structurally related. The products were the \\ result of the click reaction of three cores and four different azides in tetrahydrofuran applying \\ a homogeneous copper catalysis. The reaction intermediates and products were obtained in very good \\ to excellent yields using straightforward and simple work-up procedures. This new family of compounds \\ contain electroactive moieties such as carbazole and triphenylamine which may turn them into excellent \\ candidates for the development of optoelectronic organic materials.
}

Received 28th August 2017

Accepted 4th October 2017

DOI: $10.1039 / \mathrm{c} 7 \mathrm{ra09558a}$

rsc.li/rsc-advances also generated an explosive growth of click chemistry as a valuable concept in materials science. The replacement of the original method for one that uses simple and robust chemistry, avoiding harsh conditions, rapidly attracted the interest of the community of scientists belonging to the field of macromolecular and material science. After the first implementation in 2004 of the copper(I) catalyzed reaction in dendrimer synthesis, ${ }^{6}$ CuAAC has been widely used to design and prepare polymeric structures with macromolecular architectures which proved to be very difficult or almost impossible to get before. Dendrimer synthesis often requires excess of reactants, high cost catalysts and tiresome chromatographic separations with the generation of considerable waste. The possibility of high efficiency and easy purification without excess of reactants make CuAAC very attractive for the synthesis of dendrimers and after 2010 several review articles describe the application of CuAAC in dendrimers synthesis or functionalization. ${ }^{7}$

Triazole unit is a non-natural heterocycle and its origin is from chemical synthesis and the copper-catalyzed 1,3-dipolar cycloaddition is an orthogonal coupling reaction which is widely tolerated for several functional groups that allowed to obtain this type of ring. ${ }^{8}$ This scaffold has been widely used in many areas such as biology, drug design, supramolecular chemistry, nano-optoelectronics, macromolecular and materials sciences. Particularly in the last two applications, the triazole ring acts simply as linkage. ${ }^{9}$ In organometallics chemistry, $\mathrm{N}(2)$ and $\mathrm{N}(3)$ of the triazole can coordinate with different metals when the macromolecules contain some spatially closed triazole rings. ${ }^{10}$ Many new materials have been successfully obtained by CuAAC, but the most intriguingly aspect is the nature and the function of the 1,2,3-triazole structure within the resulting material properties. This aromatic ring often affects the properties of the molecules to which it belongs. For example, triazole $\pi$-system provides aromatic character for monomers, conjugated polymers and fluorescent probes. ${ }^{11}$
Instituto de Química Rosario, Facultad de Ciencias Bioquímicas y Farmacéuticas, Universidad Nacional de Rosario - CONICET, Suipacha 531, S2002RLK Rosario, Argentina. E-mail: mangione@iquir-conicet.gov.ar

$\dagger$ Electronic supplementary information (ESI) available: Detailed synthetic procedures and complete characterization data are depicted in ESI for each compound described in Schemes 1 and 2. See DOI: 10.1039/c7ra09558a 


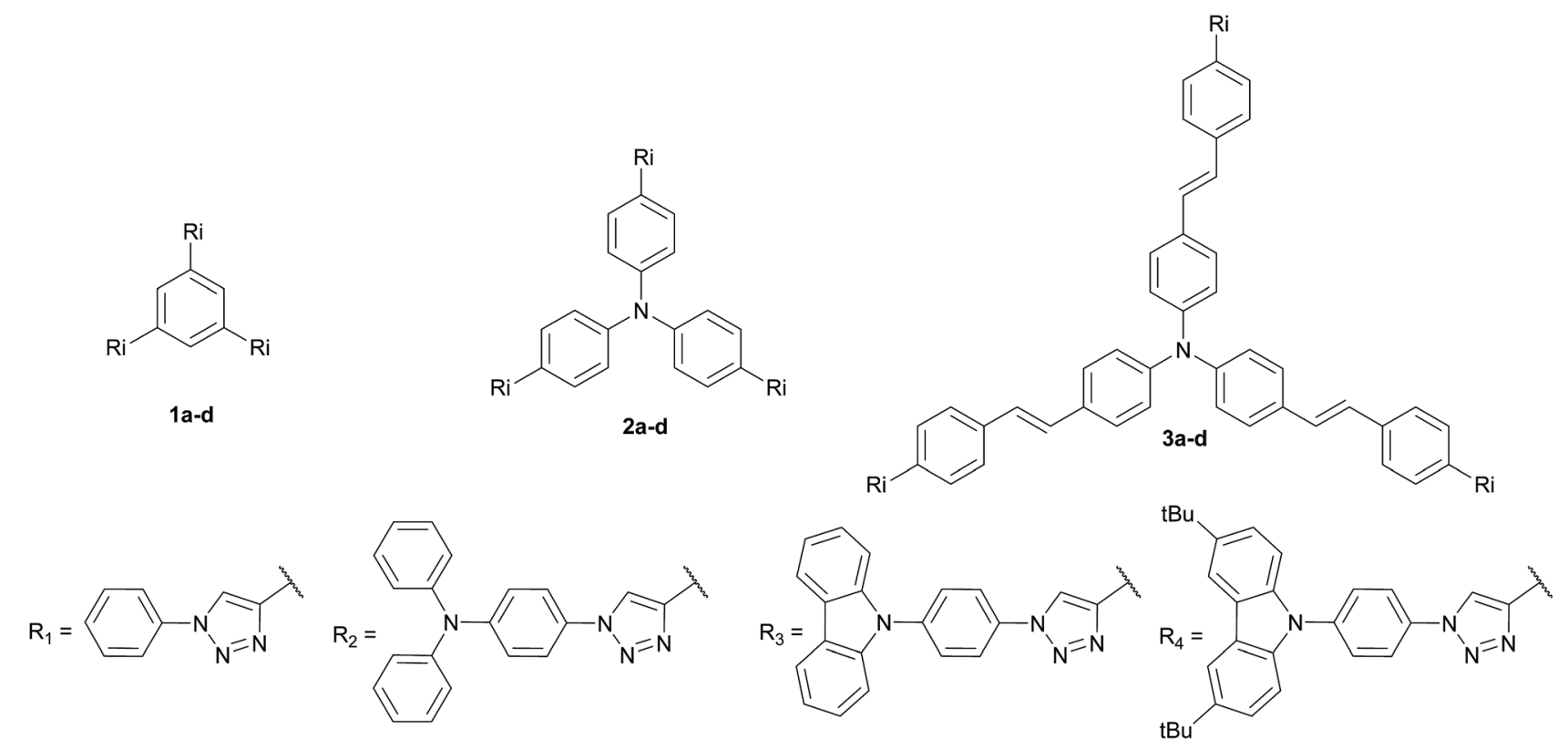

Fig. 1 Cores and dendrons of triazolic dendrimers.

On the other hand, the triarylamine core has been widely used in the development of electro-optical materials such as organic light emitting diodes (OLEDs), organic field-effect transistors (OFETs), non-linear materials and xerography, due to its good electron donating and transporting capabilities. Furthermore when it is used as core gives rise to star-shape molecular architectures. ${ }^{12}$

Hence, the electronic characteristics of triarylamines combined with the interesting properties that the triazole moiety can confer to polymers and dendrimers, such as solubility, swelling, metal adhesion, macromolecular architecture, among others, makes the CuAAC reaction a convenient tool for the development of novel dendritic organic materials.

Herein, we describe the design and synthesis of structurally related families of triazole derived dendrimers (Fig. 1). These families of compounds were efficiently synthesized in high yields, with no need for chromatographic separations. The new set of macromolecules with electroactive moieties such as triphenylamine and carbazole are promising targets for optoelectronic applications.

\section{Experimental}

\section{General considerations}

Melting points were taken on a Leitz Wetzlar Microscope Heating Stage, Model 350 apparatus and are uncorrected. ${ }^{1} \mathrm{H}$ and ${ }^{13} \mathrm{C}$ NMR spectra were recorded on Bruker Avance-300 spectrometer with $\mathrm{Me}_{4} \mathrm{Si}$ as the internal standard and dimethylsulfoxide- $d 6$ or chloroform- $d$ as solvents. Abbreviations: $\mathrm{s}=$ singlet, $\mathrm{d}=$ doublet, $\mathrm{t}=$ triplet, and $\mathrm{m}=$ multiplet expected but not resolved. Mass spectra of dendrons were recorded in a Shimadzu QP2010 Plus instrument, ion source temperature = $300{ }^{\circ} \mathrm{C}$, and detector voltage $=70 \mathrm{kV}$. High resolution mass spectra of the dendrimers were analyzed by electrospray ionization (ESI) using a Bruker micrOTOF-Q II instrument, positive ion polarity or by ultraviolet matrix assisted laser desorption-ionization mass spectrometry (UV-MALDI MS) and by ultraviolet laser desorption-ionization mass spectrometry (UV-LDI MS) performed on the Bruker Ultraflex Daltonics TOF/ TOF mass spectrometer. Mass spectra were acquired in linear positive and negative ion modes. Stock solutions of samples were prepared in chloroform. External mass calibration was made using $\beta$-cyclodextrin (MW 1134) with nHo as matrix in positive and negative ion mode. Sample solutions were spotted on a MTP 384 target plate polished steel from Bruker Daltonics (Leipzig, Germany). For UV-MALDI MS matrix solution was prepared by dissolving GA (gentisic acid, $1 \mathrm{mg} \mathrm{mL}^{-1}$ ) in water and dry droplet sample preparation was used according to Nonami $e t$ al. ${ }^{13}$ loading successively $0.5 \mu \mathrm{L}$ of matrix solution, analyte solution and matrix solution after drying each layer at normal atmosphere and room temperature. For UV-LDI MS experiments two portions of analyte solution $(0.5 \mu \mathrm{L} \times 2)$ were loaded on the probe and dried successively (two dry layers). Desorption/ionization was obtained by using the frequencytripled Nd:YAG laser $(355 \mathrm{~nm})$. The laser power was adjusted to obtain high signal-to-noise ratio $(\mathrm{S} / \mathrm{N})$ while ensuring minimal fragmentation of the parent ions and each mass spectrum was generated by averaging 100 lasers pulses per spot. Spectra were obtained and analyzed with the programs FlexControl and FlexAnalysis, respectively. Reactions were monitored by TLC on $0.25 \mathrm{~mm}$ E. Merck Silica Gel Plates (60F254), using UV light $(254 \mathrm{~nm})$ and phosphomolybdic acid as developing agent. Flash column chromatographies using E. Merck silica gel $60 \mathrm{H}$ were performed by gradient elution of mixture of $n$-hexane and increasing volumes of dichloromethane or ethyl acetate. Reactions were run under an argon atmosphere with freshly anhydrous distilled solvents, unless otherwise noted. 
Yields refer to chromatographically and spectroscopically homogeneous materials, unless otherwise stated.

General procedure for the synthesis of the triazolic dendrimers are described, the synthetic protocols for the preparation of cores 4, 5 and 6 and dendrons 7, 8, 9 and 10 are fully described in the ESI. $\dagger$

\section{General procedure for the synthesis of the dendrimers 1a-d, 2a-d and 3a-d}

Core $(0.12 \mathrm{mmol})$ was dissolved in anhydrous tetrahydrofuran ( $4 \mathrm{mM}$ ) under Ar atmosphere. Azide (3.6 equiv.), $\mathrm{Cu}\left(\mathrm{PPh}_{3}\right)_{3} \mathrm{Br}$ (21\% mole) and $N, N$-di-isopropylethylamine (3 equiv.) were added and the reaction was refluxed. The completion of the reaction was controlled by TLC until consumption of the cores. Solvent was evaporated and the resulting crude product suspended in ethanol. The solid was filtered, washed with ethanol and suspended in methyl-tert-butyl ether. Solid was filtered again and dried affording pure dendrimer as coloured solid. Numbering of each structure is arbitrary and is described in the ESI. $\dagger$

Dendrimer 1a. Yield: (51.4 mg, 81\%); light yellow solid (ethanol, MTBE); mp 296-299 ${ }^{\circ} \mathrm{C}$. IR: $1597(\mathrm{C}=\mathrm{N}), 1507(\mathrm{C}=\mathrm{C})$, 1234, 1043, $756(\mathrm{Ph}), 685(\mathrm{Ph}) \mathrm{cm}^{-1}$. ${ }^{1} \mathrm{H}-\mathrm{NMR}$ (300 MHz, DMSO$\left.\mathrm{d}_{6}\right): \delta=7.05\left(6 \mathrm{H}, \mathrm{dd}, J_{1}=J_{2}=7.8 \mathrm{~Hz}, 7-\mathrm{H}\right), 7.53(3 \mathrm{H}, \mathrm{dd}, J=$ $7.4 \mathrm{~Hz}, 8-\mathrm{H}), 8.026 \mathrm{H},(6 \mathrm{H}, \mathrm{d}, J=8.2 \mathrm{~Hz}, 6-\mathrm{H}), 8.57$ (3H, s, 2-H), 9.49 (3H, s, 4-H vinylic). ${ }^{13} \mathrm{C}-\mathrm{NMR}\left(75 \mathrm{MHz}, \mathrm{DMSO}-\mathrm{d}_{6}\right): \delta=120.0$ (C-6), 120.3 (C-4), 122.0 (C-2), 128.8 (C-8), 130.0 (C-7), 131.8 (C1), 136.6 (C-5), 146.9 (C-3). MALDI-TOF ( $\mathrm{m} / \mathrm{z})$ : calcd for $\mathrm{C}_{30} \mathrm{H}_{21} \mathrm{~N}_{9} \mathrm{Na}^{+} 530.2\left(\mathrm{M}+\mathrm{Na}^{+}\right)$: obsd, 530.1.

Dendrimer 1b. Yield: $115.5 \mathrm{mg}$ (95\%); gray solid (ethanol, MTBE); mp 190-193 ${ }^{\circ} \mathrm{C}$. IR: $1512(\mathrm{C}=\mathrm{N}), 1450(\mathrm{C}=\mathrm{C}), 1227$, 1036, $748(\mathrm{Ph}), 721(\mathrm{Ph}) \mathrm{cm}^{-1} .{ }^{1} \mathrm{H}-\mathrm{NMR}$ (300 MHz, DMSO-d 6 ): $\delta=7.09(12 \mathrm{H}, \mathrm{d}, J=8.3 \mathrm{~Hz}, 10-\mathrm{H}), 7.12(6 \mathrm{H}, \mathrm{d}, J=7.4 \mathrm{~Hz}, 12-\mathrm{H})$, $7.14(6 \mathrm{H}, \mathrm{d}, J=9.0 \mathrm{~Hz}, 6-\mathrm{H}), 7.35(12 \mathrm{H}, \mathrm{dd}, J=8.3, J=7.4 \mathrm{~Hz}$, $11-\mathrm{H}), 7.87(6 \mathrm{H}, \mathrm{d}, J=8.9 \mathrm{~Hz}, 7-\mathrm{H}), 8.51(3 \mathrm{H}, \mathrm{s}, 2-\mathrm{H}), 9.36(3 \mathrm{H}, \mathrm{s}$, 4-H vinylic). ${ }^{13} \mathrm{C}-\mathrm{NMR}$ (75 MHz, DMSO-d 6 ): $\delta=119.9$ (C-4), 121.4 (C-7), 121.8 (C-2), 122.9 (C-6), 123.8 (C-12), 124.6 (C-10), 129.8 (C-11), 130.7 (C-5), 131.8 (C-1), 146.6 (C-3), 146.7 (C-9), 147.7 (C8). MALDI-TOF $(\mathrm{m} / \mathrm{z})$ : calcd for $\mathrm{C}_{66} \mathrm{H}_{42} \mathrm{~N}_{12} \mathrm{H}^{+} 1009.4\left(\mathrm{M}+\mathrm{H}^{+}\right)$: obsd, 1009.9.

Dendrimer 1c. Yield: $109.1 \mathrm{mg}$ (84\%); ocher solid (ethanol, MTBE); $\mathrm{mp}>300{ }^{\circ} \mathrm{C}$. IR: $1589(\mathrm{C}=\mathrm{N}), 1514(\mathrm{C}=\mathrm{C}), 1487,1271$, 1036, $752(\mathrm{Ph}), 649(\mathrm{Ph}) \mathrm{cm}^{-1}$. H-NMR (300 MHz, DMSO-d $\left.{ }_{6}\right): \delta=$ $7.33(6 \mathrm{H}, \mathrm{ddd}, J=7.7, J=2.1 \mathrm{~Hz}, 12-\mathrm{H}), 7.46-7.50(12 \mathrm{H}, \mathrm{m}, 10-$ $\mathrm{H}, 11-\mathrm{H}), 7.95(6 \mathrm{H}, \mathrm{d}, J=8.7 \mathrm{~Hz}, 6-\mathrm{H}), 8.28(6 \mathrm{H}, \mathrm{d}, J=9.7 \mathrm{~Hz}, 13-$ H), $8.35(6 \mathrm{H}, \mathrm{d}, J=8.6 \mathrm{~Hz}, 7-\mathrm{H}), 8.68(3 \mathrm{H}, \mathrm{s}, 2-\mathrm{H}), 9.70(3 \mathrm{H}, \mathrm{s}, 4-\mathrm{H}$ vinylic). ${ }^{13} \mathrm{C}-\mathrm{NMR}$ (75 MHz, DMSO-d 6 ): $\delta=109.7$ (C-10), 120.4 (C-12), 120.4 (C-4), 120.6 (C-13), 121.7 (C-7), 122.1 (C-2), 122.9 (C-14), 126.4 (C-11), 128.2 (C-6), 131.8 (C-1), 135.4 (C-8), 137.0 (C-5), 140.0 (C-9), 147.0 (C-3). MALDI-TOF $(\mathrm{m} / \mathrm{z})$ : calcd for $\mathrm{C}_{66} \mathrm{H}_{42} \mathrm{~N}_{12} \mathrm{Na}^{+}$1025.4 $\left(\mathrm{M}+\mathrm{Na}^{+}\right)$: obsd, 1025.9.

Dendrimer 1d. Yield: $120.5 \mathrm{mg}(90 \%)$; yellow solid (ethanol, MTBE); mp 240-242 ${ }^{\circ} \mathrm{C}$. IR: $2957\left(\mathrm{C}-\mathrm{H}^{t} \mathrm{Bu}\right), 1520(\mathrm{C}=\mathrm{N}), 1489$ $(\mathrm{C}=\mathrm{C}), 1364,1261,1036,810(\mathrm{Ph}) \mathrm{cm}^{-1}$. H-NMR $(300 \mathrm{MHz}$, $\left.\mathrm{CDCl}_{3}\right): \delta=1.47\left(54 \mathrm{H}, \mathrm{s}, \mathrm{CH}_{3}\right), 7.41(6 \mathrm{H}, \mathrm{d}, J=8.5 \mathrm{~Hz}, 10-\mathrm{H})$, $7.48(6 \mathrm{H}, \mathrm{dd}, J=8.7, J=1.9 \mathrm{~Hz}, 11-\mathrm{H}), 7.79(6 \mathrm{H}, \mathrm{d}, J=8.8 \mathrm{~Hz}, 6-$
$\mathrm{H}), 8.06(6 \mathrm{H}, \mathrm{d}, J=8.8 \mathrm{~Hz}, 7-\mathrm{H}), 8.16(6 \mathrm{H}, \mathrm{d}, J=1.4 \mathrm{~Hz}, 13-\mathrm{H})$, 8.56 (3H, s, 4-H vinylic), $8.58(3 \mathrm{H}, \mathrm{s}, 2-\mathrm{H}) .{ }^{13} \mathrm{C}-\mathrm{NMR}(75 \mathrm{MHz}$, $\left.\mathrm{CDCl}_{3}\right): \delta=32.0\left(\mathrm{CH}_{3}\right), 34.8\left(\mathrm{CMe}_{3}\right), 109.0(\mathrm{C}-10), 116.4(\mathrm{C}-13)$, 118.4 (C-4), 121.8 (C-7), 122.8 (C-2), 123.7 (C-14), 123.9 (C-11), 127.7 (C-6), 131.6 (C-1), 135.0 (C-8), 138.8 (C-9), 138.9 (C-5), 143.5 (C-12), 147.8 (C-3). MALDI-TOF (m/z): calcd for $\mathrm{C}_{90} \mathrm{H}_{90} \mathrm{~N}_{12}{ }^{+} 1338.7\left(\mathrm{M}^{+}\right)$: obsd, 1338.4.

Dendrimer 2a. Yield: $77.4 \mathrm{mg}$ (96\%); light yellow solid (ethanol, MTBE); mp 160-163 ${ }^{\circ} \mathrm{C}$. IR: $1489(\mathrm{C}=\mathrm{N}, \mathrm{C}=\mathrm{C}), 1040$, $758(\mathrm{Ph}) \mathrm{cm}^{-1} .{ }^{1} \mathrm{H}-\mathrm{NMR}\left(300 \mathrm{MHz}, \mathrm{DMSO}-\mathrm{d}_{6}\right): \delta=7.22(6 \mathrm{H}, \mathrm{d}, J$ $=8.6 \mathrm{~Hz}, 2-\mathrm{H}), 7.50(3 \mathrm{H}, \mathrm{dd}, J=7.4 \mathrm{~Hz}, 10-\mathrm{H}), 7.63(6 \mathrm{H}, \mathrm{dd}, J=$ $8.3, J=7.5 \mathrm{~Hz}, 9-\mathrm{H}), 7.91(6 \mathrm{H}, \mathrm{d}, J=8.6 \mathrm{~Hz}, 3-\mathrm{H}), 7.95(6 \mathrm{H}, \mathrm{d}, J=$ $8.0 \mathrm{~Hz}, 8-\mathrm{H}), 9.25$ (3H, s, 6-H vinylic). ${ }^{13} \mathrm{C}-\mathrm{NMR}$ (75 MHz, DMSO$\left.\mathrm{d}_{6}\right): \delta=119.0(\mathrm{C}-6), 119.9(\mathrm{C}-8), 124.2(\mathrm{C}-2), 125.2$ (C-4), 126.7 (C3), 128.7 (C-10), 129.9 (C-9), 136.6 (C-7), 146.7 (C-1), 147.1 (C-5). MALDI-TOF $(\mathrm{m} / \mathrm{z})$ : calcd for $\mathrm{C}_{42} \mathrm{H}_{30} \mathrm{~N}_{10}{ }^{+} 674.3\left(\mathrm{M}^{+}\right)$: obsd, 674.1.

Dendrimer 2b. Yield: $109.9 \mathrm{mg}$ (85\%); light green solid (ethanol, MTBE); mp 194-197 ${ }^{\circ} \mathrm{C}$. IR: $1522(\mathrm{C}=\mathrm{N}), 1491(\mathrm{C}=\mathrm{C})$, 1227, 1032, $835(\mathrm{Ph}), 748(\mathrm{Ph}) \mathrm{cm}^{-1}$. H-NMR (300 MHz, DMSO$\left.\mathrm{d}_{6}\right): \delta=7.05-7.09(18 \mathrm{H}, \mathrm{m}, 12-\mathrm{H}, 14-\mathrm{H}), 7.11(6 \mathrm{H}, \mathrm{d}, J=9.0 \mathrm{~Hz}$, 9-H), $7.18(6 \mathrm{H}, \mathrm{d}, J=8.1 \mathrm{~Hz}, 2-\mathrm{H}), 7.34(12 \mathrm{H}, \mathrm{dd}, J=7.8 \mathrm{~Hz}, J=$ $7.4 \mathrm{~Hz}, 13-\mathrm{H}), 7.81(6 \mathrm{H}, \mathrm{d}, J=8.6 \mathrm{~Hz}, 8-\mathrm{H}), 7.88(6 \mathrm{H}, \mathrm{d}, J=$ $8.2 \mathrm{~Hz}, 3-\mathrm{H}), 9.13$ (3H, s, 6-H vinylic). ${ }^{13} \mathrm{C}-\mathrm{NMR}$ (75 MHz, DMSO$\left.\mathrm{d}_{6}\right): \delta=118.8$ (C-6), 121.3 (C-8), 123.0 (C-14), 123.8 (C-9), 124.5 (C-2) 124.5 (C-12), 125.2 (C-4), 126.7 (C-3), 129.7 (C-13), 130.8 (C7), 146.6 (C-1), 146.7 (C-5), 146.7 (C-11), 147.6 (C-10). ESI $(\mathrm{m} / \mathrm{z})$ : calcd for $\mathrm{C}_{78} \mathrm{H}_{58} \mathrm{~N}_{13} \mathrm{H}^{+} 1176.4933\left(\mathrm{M}+\mathrm{H}^{+}\right)$: obsd, 1176.4925 .

Dendrimer 2c. Yield: $91.3 \mathrm{mg}$ (65\%); light orange solid (ethanol, MTBE); mp 252-255 ${ }^{\circ} \mathrm{C}$. IR: $1589(\mathrm{C}=\mathrm{N}), 1489(\mathrm{C}=\mathrm{C})$, 1281, 1032, $835(\mathrm{Ph}), 752(\mathrm{Ph}), 694(\mathrm{Ph}) \mathrm{cm}^{-1}$. H-NMR (300 MHz, DMSO-d 6 ): $\delta=7.25-7.35(12 \mathrm{H}, \mathrm{m}, 2-\mathrm{H}, 13-\mathrm{H}), 7.46(12 \mathrm{H}, \mathrm{bd}, J=$ $3.6 \mathrm{~Hz}, 8-\mathrm{H}, 12-\mathrm{H}), 7.91(6 \mathrm{H}, \mathrm{d}, J=8.6 \mathrm{~Hz}, 15-\mathrm{H}), 7.97(6 \mathrm{H}, \mathrm{d}, J=$ $8.5 \mathrm{~Hz}, 3-\mathrm{H}), 8.26$ (12H, bd, $J=8.1 \mathrm{~Hz}, 9-\mathrm{H}, 14-\mathrm{H}), 9.42(3 \mathrm{H}, \mathrm{s}, 6-$ $\mathrm{H}$ vinylic). ${ }^{13} \mathrm{C}-\mathrm{NMR}$ (75 MHz, DMSO-d $\left.\mathrm{d}_{6}\right): \delta=109.7$ (C-12), 119.2 (C-6), 120.4 (C-7), 120.6 (C-13), 121.5 (C-14), 122.9 (C-8), 124.3 (C-2), 125.1 (C-4), 126.4 (C-9), 126.8 (C-3), 128.2 (C-15), 135.4 (C11), 136.9 (C-10), 140.0 (C-16), 146.7 (C-1), 147.3 (C-5). ESI $(\mathrm{m} / \mathrm{z})$ : calcd for $\mathrm{C}_{78} \mathrm{H}_{52} \mathrm{~N}_{13}{ }^{+} 1170.4463\left(\mathrm{M}+\mathrm{H}^{+}\right)$: obsd, 1170.4460 .

Dendrimer 2d. Yield: $129.7 \mathrm{mg}$ (86\%); yellow solid (ethanol, MTBE); mp 251-254 ${ }^{\circ} \mathrm{C}$. IR: $2955\left({ }^{t} \mathrm{Bu}\right), 1522(\mathrm{C}=\mathrm{N}), 1489(\mathrm{C}=$ C), 1364, 1261, 1032, $810(\mathrm{Ph}) \mathrm{cm}^{-1} \cdot{ }^{1} \mathrm{H}-\mathrm{NMR}\left(300 \mathrm{MHz}, \mathrm{CDCl}_{3}\right)$ : $\delta=1.48\left(54 \mathrm{H}, \mathrm{s}, \mathrm{CH}_{3}\right), 7.32(6 \mathrm{H}, \mathrm{d}, 2-\mathrm{H}), 7.40(6 \mathrm{H}, \mathrm{d}, J=8.6 \mathrm{~Hz}$, $12-\mathrm{H}), 7.50$ (6H, dd, $J=8.7, J=1.9 \mathrm{~Hz}, 13-\mathrm{H}), 7.77(6 \mathrm{H}, \mathrm{d}, J=$ $8.8 \mathrm{~Hz}, 8-\mathrm{H}), 7.90(6 \mathrm{H}, \mathrm{d}, J=8.6 \mathrm{~Hz}, 3-\mathrm{H}), 8.04(6 \mathrm{H}, \mathrm{d}, J=8.8 \mathrm{~Hz}$, 9-H), 8.16 (6H, d, $J=1.6 \mathrm{~Hz}, 15-\mathrm{H}), 8.27$ (3H, s, 6- H vinylic). ${ }^{13} \mathrm{C}-$ NMR (75 MHz, $\left.\mathrm{CDCl}_{3}\right): \delta=32.0\left(\mathrm{CH}_{3}\right), 34.8\left(\mathrm{CMe}_{3}\right), 109.0(\mathrm{C}-12)$, 116.5 (C-15), 117.1 (C-6), 121.9 (C-9), 123.7 (C-16), 123.8 (C-13), 124.7 (C-2), 125.1 (C-4), 127.1 (C-3), 127.8 (C-8), 135.3 (C-10), 138.7 (C-7), 138.9 (C-11), 143.5 (C-14), 147.5 (C-1), 148.3 (C-5). ESI $(\mathrm{m} / \mathrm{z})$ : calcd for $\mathrm{C}_{102} \mathrm{H}_{100} \mathrm{~N}_{13}{ }^{+} 1506.8219\left(\mathrm{M}+\mathrm{H}^{+}\right)$: obsd, 1506.8189.

Dendrimer 3a. Yield: $62.5 \mathrm{mg}$ (77\%); light yellow solid (ethanol, MTBE); mp 187-190 ${ }^{\circ} \mathrm{C}$. IR: $1593(\mathrm{C}=\mathrm{N}), 1502(\mathrm{C}=\mathrm{C})$, 1034, $833(\mathrm{Ph}), 756(\mathrm{Ph}), 687(\mathrm{Ph}) \mathrm{cm}^{-1} .{ }^{1} \mathrm{H}-\mathrm{NMR}(300 \mathrm{MHz}$, DMSO-d $\left.{ }_{6}\right): \delta=7.07(6 \mathrm{H}, \mathrm{d}, J=8.3 \mathrm{~Hz}, 2-\mathrm{H}), 7.20(3 \mathrm{H}, \mathrm{d}, J=$ $17.0 \mathrm{~Hz}, 6-\mathrm{H}), 7.32(3 \mathrm{H}, \mathrm{d}, J=16.0 \mathrm{~Hz}, 5-\mathrm{H}), 7.52(3 \mathrm{H}, \mathrm{d}, J=$ $8.0 \mathrm{~Hz}, 16-\mathrm{H}), 7.59$ (6H, d, $J=8.5 \mathrm{~Hz}, 3-\mathrm{H}), 7.64(6 \mathrm{H}, \mathrm{d}, J=$ 
$7.7 \mathrm{~Hz}, 8-\mathrm{H}), 7.71(6 \mathrm{H}, \mathrm{d}, J=8.6 \mathrm{~Hz}, 14-\mathrm{H}), 7.95(12 \mathrm{H}, \mathrm{bd}, J=$ $7.8 \mathrm{~Hz}, 9-\mathrm{H}, 15-\mathrm{H}), 9.31$ (3H, s, 12-H vinylic). ${ }^{13} \mathrm{C}-\mathrm{NMR}(75 \mathrm{MHz}$, DMSO-d ${ }_{6}$ ): $\delta=119.5$ (C-12), 120.0 (C-9), 123.9 (C-2), 125.6 (C-14), 126.7 (C-6), 126.9 (C-15), 127.8 (C-3), 128.1 (C-5), 128.7 (C-16), 129.1 (C-13), 129.9 (C-8), 132.0 (C-4), 136.6 (C-10), 137.2 (C-7), 146.1 (C-1), 147.1 (C-11). MALDI-TOF $(\mathrm{m} / \mathrm{z})$ : calcd for $\mathrm{C}_{66} \mathrm{H}_{49} \mathrm{~N}_{10}{ }^{+}$981.4 $\left(\mathrm{M}+\mathrm{H}^{+}\right)$: obsd, 981.3.

Dendrimer 3b. Yield: $133.9 \mathrm{mg}$ (90\%); yellow solid (ethanol, MTBE); mp 181-184 ${ }^{\circ} \mathrm{C}$. IR: $1593(\mathrm{C}=\mathrm{N}), 1506(\mathrm{C}=\mathrm{C}), 1315$, 1227, $841(\mathrm{Ph}), 748(\mathrm{Ph}) \mathrm{cm}^{-1} .{ }^{1} \mathrm{H}-\mathrm{NMR}$ (300 MHz, DMSO-d 6 ): $\delta=7.03-7.13(30 \mathrm{H}, \mathrm{m}, 2-\mathrm{H}, 15-\mathrm{H}, 18-\mathrm{H}, 20-\mathrm{H}), 7.18(3 \mathrm{H}, \mathrm{d}, J=$ $16.9 \mathrm{~Hz}, 5-\mathrm{H}$ vinylic), 7.29 (3H, d, $J=16.5 \mathrm{~Hz}, 6-\mathrm{H}$ vinylic), 7.35 (12H, dd, $J=7.9 \mathrm{~Hz}, 19-\mathrm{H}), 7.57$ (6H, d, $J=8.2 \mathrm{~Hz}, 3-\mathrm{H}), 7.68$ $(6 \mathrm{H}, \mathrm{d}, J=8.3 \mathrm{~Hz}, 8-\mathrm{H}), 7.80(6 \mathrm{H}, \mathrm{d}, J=8.8 \mathrm{~Hz}, 14-\mathrm{H}), 7.92(6 \mathrm{H}$, $\mathrm{d}, J=8.2 \mathrm{~Hz}, 9-\mathrm{H}), 9.18\left(3 \mathrm{H}, \mathrm{s}, 12-\mathrm{H}\right.$ triazole). ${ }^{13} \mathrm{C}-\mathrm{NMR}(75 \mathrm{MHz}$, DMSO-d ${ }_{6}$ ): $\delta=119.5$ (C-12), 121.4 (C-14), 123.0 (C-15), 123.8 (C2), 123.8 (C-20), 125.5 (C-18), 126.9 (C-6 vinylic), 126.9 (C-8), 127.8 (C-3), 128.1 (C-5 vinylic), 129.2 (C-10), 129.8 (C-19), 130.8 (C-13), 132.0 (C-4), 137.1 (C-7), 146.1 (C-1), 146.7 (C-17), 146.9 (C-11), 147.6 (C-16). MALDI-TOF $(\mathrm{m} / \mathrm{z})$ : calcd for $\mathrm{C}_{102} \mathrm{H}_{76} \mathrm{~N}_{13}{ }^{+}$ 1482.8 $\left(\mathrm{M}+\mathrm{H}^{+}\right)$: obsd, 1482.3.

Dendrimer 3c. Yield: $74.1 \mathrm{mg}$ (51\%); yellow solid (ethanol, MTBE); mp 198-201 ${ }^{\circ} \mathrm{C}$. IR: $1591(\mathrm{C}=\mathrm{N}), 1505(\mathrm{C}=\mathrm{C}), 1273$, 1031, $833(\mathrm{Ph}), 752(\mathrm{Ph}), 696(\mathrm{Ph}) \mathrm{cm}^{-1} \cdot{ }^{1} \mathrm{H}-\mathrm{NMR}(300 \mathrm{MHz}$, DMSO- $\left.\mathrm{d}_{6}\right): \delta=7.08(6 \mathrm{H}, \mathrm{d}, J=7.6 \mathrm{~Hz}, 2-\mathrm{H}), 7.24-7.34(12 \mathrm{H}$, $\mathrm{m}, 5-\mathrm{H}, 6-\mathrm{H}$ vinylics, 20-H), $7.47(12 \mathrm{H}$, bd, $J=3.4 \mathrm{~Hz}, 18-\mathrm{H}$, $19-\mathrm{H}), 7.61(6 \mathrm{H}, \mathrm{d}, J=8.1 \mathrm{~Hz}, 3-\mathrm{H}), 7.75(6 \mathrm{H}, \mathrm{d}, J=8.0 \mathrm{~Hz}, 9-$ $\mathrm{H}), 7.92(6 \mathrm{H}, \mathrm{d}, J=8.6 \mathrm{~Hz}, 15-\mathrm{H}), 7.99(6 \mathrm{H}, \mathrm{d}, J=7.8 \mathrm{~Hz}, 8-\mathrm{H})$, $8.26(6 \mathrm{H}, \mathrm{d}, J=8.5 \mathrm{~Hz}, 14-\mathrm{H}), 8.27(6 \mathrm{H}, \mathrm{d}, J=7.4 \mathrm{~Hz}, 21-\mathrm{H})$, 9.46 (3H, s, 12-H triazole). ${ }^{13} \mathrm{C}-\mathrm{NMR}\left(75 \mathrm{MHz}, \mathrm{DMSO}-\mathrm{d}_{6}\right): \delta=$ 110.2 (C-18), 120.2 (C-12), 120.9 (C-20), 121.1 (C-21), 123.4 (C22), 124.4 (C-2), 126.2 (C-8), 126.9 (C-19), 127.5 (C-5 or C-6 vinylics), 127.5 (C-9), 128.3 (C-3), 128.7 (C-5/6 vinylics), 128.7 (C-15), 129.5 (C-7), 132.5 (C-4), 135.9 (C-13), 137.4 (C16), 137.8 (C-10), 140.5 (C-17), 146.7 (C-1), 147.8 (C-11). MALDI-TOF $(\mathrm{m} / \mathrm{z})$ : calcd for $\mathrm{C}_{102} \mathrm{H}_{70} \mathrm{~N}_{13}{ }^{+} 1476.6\left(\mathrm{M}+\mathrm{H}^{+}\right)$: obsd, 1476.3 .

Dendrimer 3d. Yield: $100.4 \mathrm{mg}$ (96\%); yellow solid (ethanol, MTBE); mp 258-260 ${ }^{\circ} \mathrm{C}$. IR: $2951\left({ }^{t} \mathrm{Bu}\right), 1593(\mathrm{C}=\mathrm{N})$, $1506(\mathrm{C}=\mathrm{C}), 1261,1032,808(\mathrm{Ph}) \mathrm{cm}^{-1} .{ }^{1} \mathrm{H}-\mathrm{NMR}(300 \mathrm{MHz}$, $\left.\mathrm{CDCl}_{3}\right): \delta=1.47\left(54 \mathrm{H}, \mathrm{s}, \mathrm{CH}_{3}\right), 7.08(3 \mathrm{H}, \mathrm{d}, J=16.2 \mathrm{~Hz}, 5-\mathrm{H})$, $7.14(6 \mathrm{H}, \mathrm{d}, J=8.6 \mathrm{~Hz}, 2-\mathrm{H}), 7.17(3 \mathrm{H}, \mathrm{d}, J=16.2 \mathrm{~Hz}, 6-\mathrm{H})$, $7.38(6 \mathrm{H}, \mathrm{d}, J=8.6 \mathrm{~Hz}, 18-\mathrm{H}), 7.47(6 \mathrm{H}, \mathrm{d}, J=8.6 \mathrm{~Hz}, 3-\mathrm{H})$, $7.49(6 \mathrm{H}, \mathrm{dd}, J=8.7, J=1.9 \mathrm{~Hz}, 19-\mathrm{H}), 7.62(6 \mathrm{H}, \mathrm{d}, J=8.5 \mathrm{~Hz}$, $8-\mathrm{H}), 7.75(6 \mathrm{H}, \mathrm{d}, J=8.8 \mathrm{~Hz}, 14-\mathrm{H}), 7.94(6 \mathrm{H}, \mathrm{d}, J=8.3 \mathrm{~Hz}, 9-$ $\mathrm{H}), 8.02(6 \mathrm{H}, \mathrm{d}, J=8.8 \mathrm{~Hz}, 15-\mathrm{H}), 8.16(6 \mathrm{H}, \mathrm{d}, J=1.5 \mathrm{~Hz}, 21-$ $\mathrm{H}), 8.29$ (3H, s, 12-H triazole). ${ }^{13} \mathrm{C}-\mathrm{NMR}\left(75 \mathrm{MHz}, \mathrm{CDCl}_{3}\right): \delta=$ $32.0\left(\mathrm{CH}_{3}\right), 34.8\left(\mathrm{CMe}_{3}\right), 109.0(\mathrm{C}-18), 116.4$ (C-21), 117.4 (C12), 121.9 (C-15), 123.7 (C-22), 123.9 (C-19), 124.3 (C-2), 126.2 (C-10), 126.9 (C-5 o C-6 vinylics), 127.0 (C-8), 127.6 (C3), 127.7 (C-14), 128.5 (C-5/6 vinylics), 129.0 (C-10), 132.1 (C-4), 135.1 (C-16), 137.8 (C-7), 138.7 (C-13), 138.9 (C-17), 143.5 (C-20), 146.8 (C-1), 148.4 (C-11). MALDI-TOF $(\mathrm{m} / \mathrm{z})$ : calcd for $\mathrm{C}_{127} \mathrm{H}_{122} \mathrm{~N}_{13} \mathrm{O}^{+} 1730.8\left(\mathrm{M}+\mathrm{MeOH}_{2}{ }^{+}{ }^{t} \mathrm{Bu}\right.$ group loss $)$ : obsd, 1730.4 .

\section{Results and discussion}

Three families of structurally related triazolic dendrimers were prepared by the combination of three different cores and four different aromatic azides, the structures of these compounds are depicted in Fig. 2.

With the exception of core 4 and dendron 7, the rest of the cores and dendrons contain electroactive moieties such as triphenylamine (cores 5 and 6, dendron 8) and carbazole (dendrons 9 and 10). The cores were synthesized from the corresponding aryl halide through a Sonogashira coupling reaction with trimethylsilylacetylene (TMSA) and further desilylation to get the free alkynes. In Scheme 1 are described the synthetic routes for every target core. The synthesis of core 4 and intermediate $\mathbf{1 5}$ has been previously reported by our laboratory. ${ }^{\mathbf{1 4}}$ There are two common steps in each synthesis: Sonogashira coupling with TMSA and basic desilylation. Core 4 was obtained after these two steps with excellent yields. Synthesis of core $\mathbf{6}$ was accomplished from commercial triphenylamine $\mathbf{1 2}$ by formylation under Vilsmeier-Haack conditions ${ }^{\mathbf{1 5}}$ in two steps furnishing tri-aldehyde derivative $\mathbf{1 3}$ in moderated yield. A Wadsworth-Horner-Emmons olefination reaction used to couple 13 with phosphonate 14 afforded tri-iodide 15 in good yield. ${ }^{\mathbf{1 6}}$ Further Sonogashira coupling reaction with TMSA followed by desilylation procedure furnished core 6 in very good yield. For the synthesis of 5, triphenylamine 12 was treated with a mixture of $\mathrm{KI}: \mathrm{KIO}_{3}$ in glacial acetic acid to give tri-iodide derivative $\mathbf{1 6}$ in moderated yield. ${ }^{17}$ After Sonogashira and desilylation reactions sequence, core 5 was obtained in good yield. ${ }^{18}$ Cores 4, 5 and 6 present three terminal alkyne functionalities arranged in a "star-shape" architecture which it will ultimately define the shaped of the resulting dendrimers.

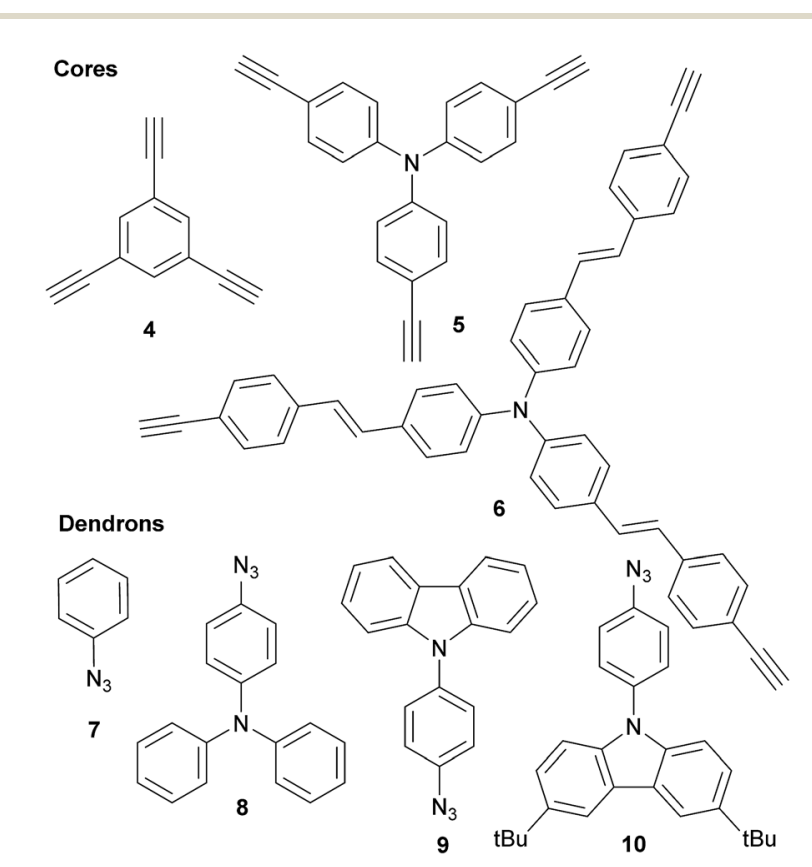

Fig. 2 Chemical structures of cores and dendrons. 


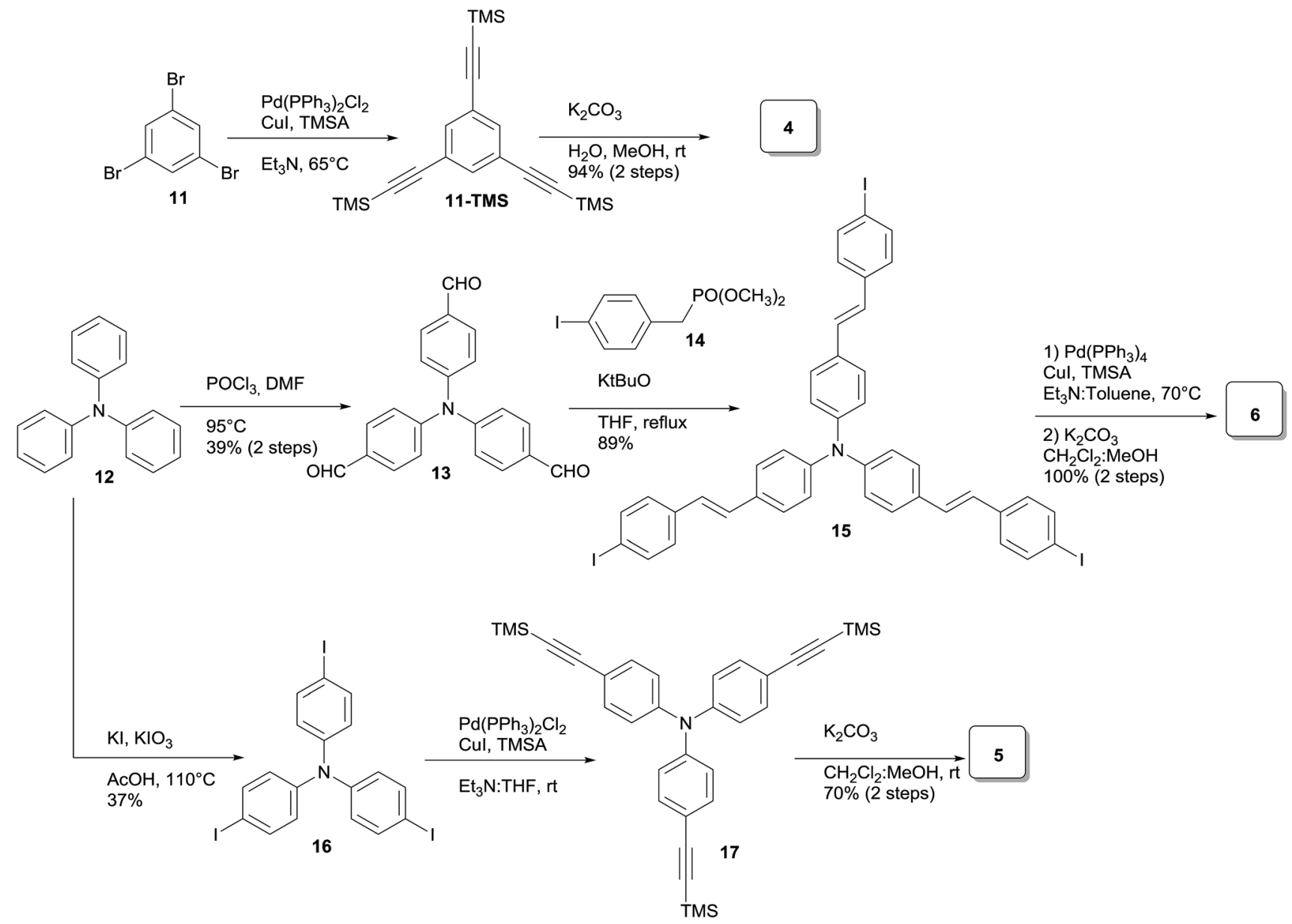

Scheme 1 Synthesis of cores.
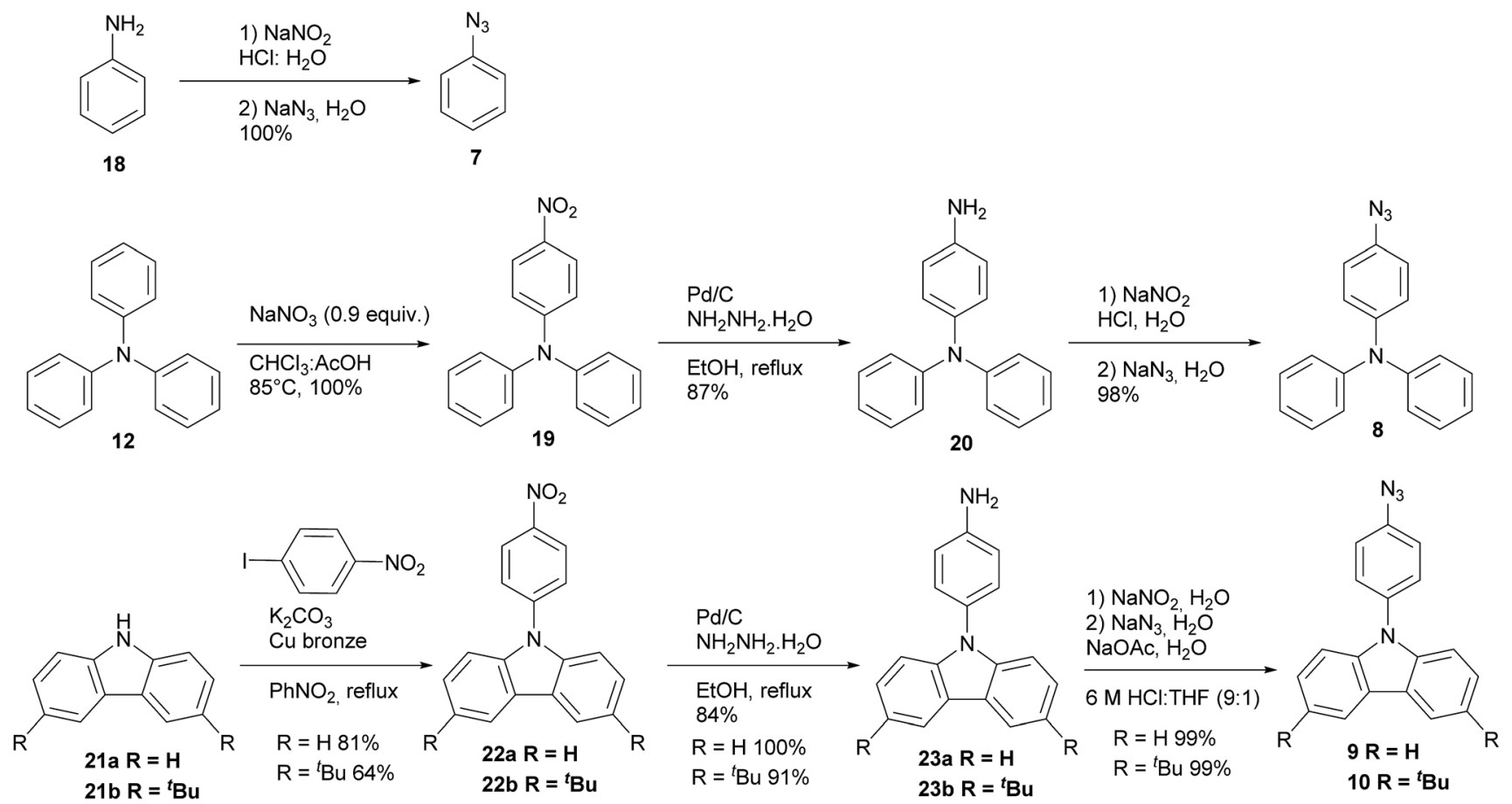

Scheme 2 Synthesis of dendrons. 
The synthesis of azides 7-10 are described in Scheme 2. All azides derivatives were obtained in very good yields through a diazotization reaction to transform the amino group into the azide one. It is worth to mention that azides showed to be highly photosensitive, therefore all the azides were used as crude products without purification and handled protected from light. Products 7, 8, 9 and 10 were characterized by ${ }^{1} \mathrm{H}$ and ${ }^{13} \mathrm{C}$ NMR and FT-IR spectroscopy. The last one was very useful to show the distinctive signal of azide group between 2160$2120 \mathrm{~cm}^{-1} \cdot{ }^{19}$ Phenyl azide 7 was prepared according to literature protocol $^{20}$ via diazotization of freshly distilled aniline 18 and further treatment with sodium azide affording 7 in excellent yield. Triphenyl azide $\mathbf{8}$ was prepared by nitration of triphenylamine $12,{ }^{21}$ the mono nitrate derivative was reduced using hydrazine monohydrate and Pd/C to furnish amine $\mathbf{2 0},{ }^{22}$ subsequently diazotization and treatment with sodium azide afforded the expected product $8 .^{21}$ The preparation of azides 9 and 10 were achieved from commercial carbazole 21a and 3,6di-tert-butyl carbazole 21b which was previously synthesized in our laboratory. ${ }^{23}$ Ullmann coupling of $21 \mathrm{a}$ and $21 \mathbf{b}^{24}$ with 4 nitroiodobenzene afforded 22a and 22b. The nitro derivatives were reduced with hydrazine ${ }^{22}$ yielding amines 23a and 23b, which were submitted to diazotization and further treatment with sodium azide to give the corresponding azides 9 and $10 .^{25}$ It is noteworthy to mention that all the reaction steps were accomplished with excellent yields and only one column chromatography purification was needed for the nitro derivatives

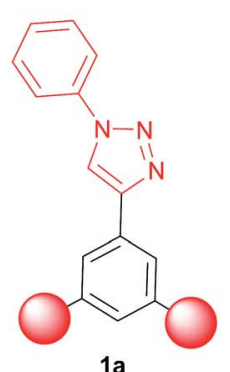

$1 \mathrm{a}$<smiles>Oc1ccc(N(c2ccc(O)cc2)c2ccc(O)cc2)cc1</smiles>

2a

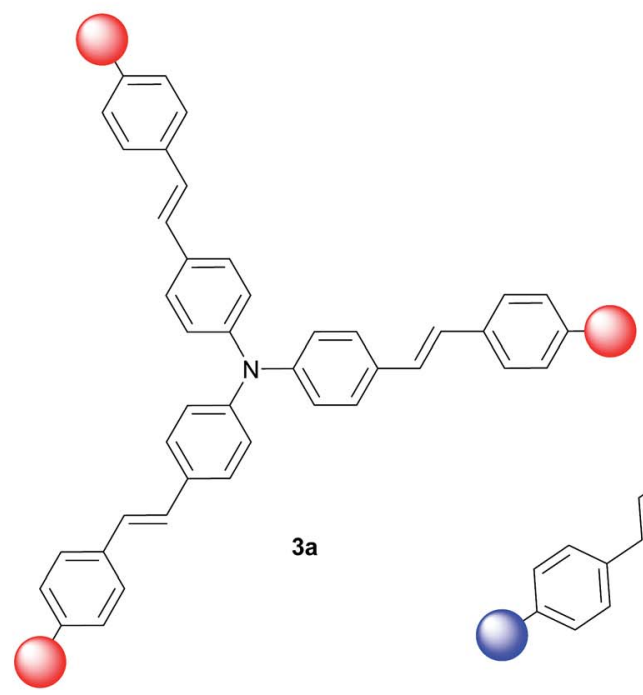

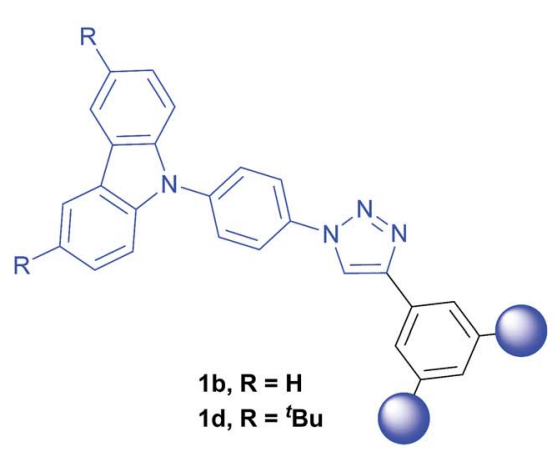

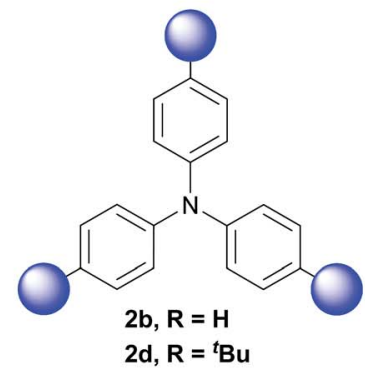

$2 \mathrm{~d}, \mathrm{R}={ }^{t_{\mathrm{Bu}}}$<smiles>[C+]=Cc1ccc(C=CC)cc1</smiles><smiles>CCc1ccc(N(c2ccccc2)c2ccc(/C=C/c3ccc(OC)cc3)cc2)cc1</smiles>

$3 \mathrm{~d}, \mathrm{R}={ }^{\mathrm{t}} \mathrm{Bu}$

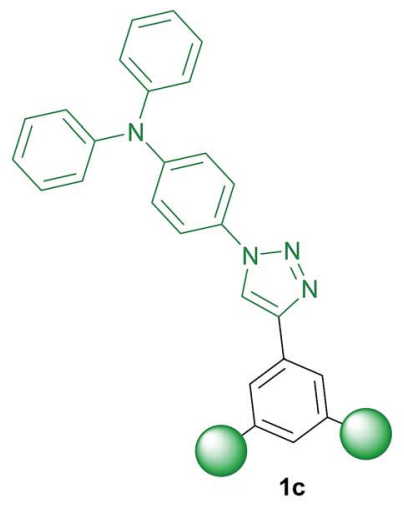<smiles>c1ccc(N(c2ccc(C3CCCCC3)cc2)c2ccc(C3CCCCC3)cc2)cc1</smiles>

2c

Fig. 3 Triazolic star-shaped dendrimers structures. 
22a and 22b, all the others compounds were solids and purified by crystallization.

Once all the components needed for the click reaction were synthesized, we looked for a general coupling condition that could allow us to attach all the cores with the corresponding azides in a convergent manner, regardless their chemical structures, to obtain triazolic dendrimers with star-shaped architecture. In a typical procedure for a CuAAC reaction, the $\mathrm{Cu}(\mathrm{I})$ cation used as catalyst was generated in situ from a copper(II) salt and a reducing agent (most often sodium ascorbate). These systems are efficient but limited to substrates with high water tolerance. ${ }^{26}$ Sometimes, in order to apply milder reaction conditions or increase the reaction applicability, addition of ligands to protect $\mathrm{Cu}(\mathrm{I})$ centers is a common strategy. In our case, the use of sodium ascorbate and $\mathrm{CuSO}_{4}$ as source of $\mathrm{Cu}(\mathrm{I})$ in different proportions, afforded mixtures of products and partial recovery of starting materials. We assumed that incomplete reactions were a consequence of only partial solubility of starting material in the aqueous solvents mixture. Hence, we looked for homogeneous conditions for the dipolar cycloadditions and we found that the used of $\mathrm{Cu}\left(\mathrm{PPh}_{3}\right)_{3} \mathrm{Br}$ and DiPEA as organic base in tetrahydrofuran allowed us to obtain the expected products in very good yields. $\mathrm{Cu}\left(\mathrm{PPh}_{3}\right)_{3} \mathrm{Br}$ is a crystalline solid, stable to air and ambient moisture and it is prepared by the addition of triphenylphosphine to a hot methanolic solution of $\mathrm{CuBr}_{2} \cdot{ }^{27}$ This procedure avoids the need to reflux moisture-sensitive $\mathrm{CuBr}$ with triphenylphosphine. Furthermore, this complex is soluble in several organic solvents and can be stored under air for prolonged times, without any visible decomposition. ${ }^{28}$ In a general procedure, 0.10-0.12 mmol of core were dissolved in THF ( 3 to $4 \mathrm{mM}$ ), azide (3.6 equivalents), 0.06 equivalents of $\mathrm{Cu}(\mathrm{I})$ catalyst and 3 equivalents of DiPEA were used to afforded the expected products showed in Fig. 3.

The twelve triazolic dendrimers (Fig. 3) can be classified in three families according to the identity of the core. For the dendrimers 1a-d the common core was benzene, 2a-d triphenylamine and 3a-d conjugated triphenylamine. All the products were obtained applying the same reaction conditions with high yields. The coloured solid dendrimers were purified by suspension of the solids in two different solvents or a mixture of both: ethanol and methyl-tert-butyl ether. This treatment allowed removing excess of azides, copper salts and any other by-product formed in the reaction and avoids the use of chromatography column step to isolate pure triazoles. All these compounds are very polar and therefore soluble in polar aprotic solvents such as dimethylsulfoxide, tetrahydrofuran and slightly soluble in dichloromethane. The last one is better to dissolve tert-butylated derivatives $\mathbf{1 d}$, 2d and 3d. As far as we know, there are not literature references related to the preparation of structurally related families of aromatic triazoles. A few of triazolic dendritic structures has been published and they showed that this type of compounds have a high potential on new material design. ${ }^{\mathbf{2 9 , 3 0}}$ Additionally, the purification procedure was simple and similar in each case with no need for column chromatography and afforded pure samples.

\section{Conclusions}

In this work we were able to synthesize twelve dendritic aromatic triazoles structurally related using four different aromatic azides as dendrons and varying the chemical identity of the core. A standard reaction procedure was developed and applied in all cases with very good results. The homogeneous CuAAC reaction afforded solid compounds and their purification only included a suspension in different washing solvents avoiding any chromatography column technique. All the dendrimers presented a starburst molecular shape and includes triarylamines as electroactive moiety which turns them into excellent candidates for the development of novel organic materials. The structural changes introduced in every family of compounds were selected in order to establish the relationship between chemical structure-optoelectronic properties. The optoelectronic studies are being carried out and will be published in due course.

\section{Conflicts of interest}

The authors declare no competing financial interest.

\section{Acknowledgements}

We thank Consejo Nacional de Investigaciones Científicas y Técnicas (CONICET - Argentina), Agencia Nacional de Promoción Científica y Tecnológica (ANPCyT - Argentina), Fundación Nuevo Banco de Santa Fe (Santa Fe - Argentina) and Universidad Nacional de Rosario. M. I. M. and R. A. S are scientific members of CONICET.

\section{Notes and references}

1 H. C. Kolb, M. G. Finn and K. B. Sharpless, Angew. Chem., 2001, 113, 2056; Angew. Chem., Int. Ed., 2001, 40, 2004.

2 J.-F. Lutz, Angew. Chem., Int. Ed., 2007, 46, 1025.

3 (a) C. Barner-Kowollik and A. J. Inglis, Macromol. Chem. Phys., 2009, 210, 987; (b) S. Mongkhontreerat, M. V. Walter, O. C. J. Andren, Y. Cai and M. Malkoch, Adv. Funct. Mater., 2015, 25, 4837; (c) X. Wang, B. Huang, X. Liu and P. Zhan, Drug Discovery Today, 2016, 21, 118; (d) E. Haldon, M. C. Nicasio and P. J. Pérez, Org. Biomol. Chem., 2015, 13, 9528.

4 (a) V. V. Rostovtsev, L. G. Green, V. V. Folkin and K. B. Sharpless, Angew. Chem., Int. Ed., 2002, 41, 2596; (b) C. W. Tornøe, M. Meldal and C. Christensen, J. Org. Chem., 2002, 67, 3057.

5 R. Huisgen, R. Knorr, L. Moebius and G. Szeimies, Chem. Ber., 1965, 98, 4014.

6 (a) P. Wu, A. K. Feldman, A. K. Nugent, C. J. Hawker, A. Scheel, B. Voit, J. Pyun, J. M. J. Fréchet, K. B. Sharpless and V. V. Folkin, Angew. Chem., 2004, 116, 4018; (b) P. Wu, A. K. Feldman, A. K. Nugent, C. J. Hawker, A. Scheel, B. Voit, J. Pyun, J. M. J. Fréchet, K. B. Sharpless and V. V. Folkin, Angew. Chem., Int. Ed., 2004, 43, 3928. 
7 (a) W. Xi, T. F. Scott, C. J. Kloxin and C. N. Bowman, Adv. Funct. Mater., 2014, 24, 2572; (b) M. Arseneault, C. Wafer and J.-F. Morin, Molecules, 2015, 20, 9263; (c) A. Marrocchi, A. Facchetti, D. Lanari, S. Santoro and L. Vaccaro, Chem. Sci., 2016, 7, 6298; (d) M. Sowinska and Z. UrbanczykLipkowska, New J. Chem., 2014, 38, 2168.

8 C.-H. Wong and S. C. Zimmerman, Chem. Commun., 2013, 49, 1679.

9 K. C. Majumdar and K. Ray, Synthesis, 2011, 3767.

10 Y. Fu, Y. Liu, X. Fu, L. Zou, H. Li, M. Li, X. Chen and J. Qin, Chin. J. Chem., 2010, 28, 2226.

11 S. S. Bag and R. Kundu, J. Org. Chem., 2011, 76, 3348.

12 Z. Ning and H. Tian, Chem. Commun., 2009, 5483.

13 H. Nonami, S. Fukui and R. Erra-Balsells, J. Mass Spectrom., 1997, 32, 287.

14 M. I. Mangione, R. A. Spanevello, A. Rumbero, D. Heredia, G. Marzari, L. Fernandez, L. Otero and F. Fungo, Macromolecules, 2013, 46, 475.

15 T. Malegol, S. Gmouh, M. A. A. Meziane, M. Blanchard-Desce and O. Mongin, Synthesis, 2005, 11, 1771.

16 (a) H. Shao, X. Chen, Z. Wang and P. Lu, J. Lumin., 2007, 127, 349; (b) H. F. Kung, P. M. Kung and Z. Zhuang, U.S. Pat., 7,297,820, 2007.

17 T. Khanasa, N. Jantasing, S. Morada, N. Leesakul, R. Tarsang, S. Namuangruk, T. Kaewin, S. Jungsuttiwong, T. Sudyoadsuk and V. Promarak, Eur. J. Org. Chem., 2013, 2608.

18 (a) S. P. McIlroy, E. Cló, L. Nikolajsen, P. K. Frederiksen, C. B. Nielsen, K. V. Mikkelsen, K. V. Gothelf and
P. R. Ogilby, J. Org. Chem., 2005, 70, 1134; (b) P. Hrobárik, V. Hrobáriková, I. Sigmundová, P. Zahradník, M. Fakis, I. Polyzos and P. Persephonis, J. Org. Chem., 2011, 76, 8726; (c) K. Vongnam, T. Vilaivan and M. Sukwattanasinitt, J. Fluoresc., 2014, 24, 197.

19 E. Lieber, C. M. Ramachandra Rao, T. S. Chao and C. W. W. Hoffman, Anal. Chem., 1939, 19, 55.

20 H. J. Lucas and E. R. Kennedy, Org. Synth., 1939, 19, 55.

21 L. C. Raiford and A. L. LeRosen, J. Am. Chem. Soc., 1944, 66, 1872.

22 (a) W. Y. Lee, T. Kurosawa, S. T. Lin, T. Higashihara, M. Ueda and W. C. Chen, Chem. Mater., 2011, 23, 4487; (b) Y. Liu, D. Chao and H. Yao, Org. Electron., 2014, 15, 1422.

23 M. I. Mangione and R. A. Spanevello, Tetrahedron Lett., 2015, $56,465$.

24 S. Tripathi, M. Mohan, R. K. Pandey and K. Misra, Indian J. Chem., Sect. B: Org. Chem. Incl. Med. Chem., 1999, 38, 317.

25 B. Schulze, D. Escudero, C. Friebe, R. Siebert, H. Görls, S. Sinn, M. Thomas, S. Mai, J. Popp, B. Dietzek, L. González and U. S. Schubert, Chem.-Eur. J., 2012, 18, 4010. 26 S. Lal and S. Diez-González, J. Org. Chem., 2011, 76, 2367. 27 J. Germain, F. Svec and J. M. J. Fréchet, Chem. Mater., 2008, 20, 7069.

28 R. Gujadhur and D. Venkataraman, Synth. Commun., 2001, 31, 2865.

29 Q. Zhang, Z. Ning and H. Tian, Dyes Pigm., 2009, 81, 80. 30 Q. Zhang, Z. Ning, Y. Yan, S. Qian and H. Tian, Macromol. Rapid Commun., 2008, 29, 193. 